\title{
Reversible posterior leukoencephalopathy syndrome in a patient with thrombotic thrombocytopenic purpura
}

\author{
Paolo Aridon - Paolo Ragonese - Maria Antonietta Mazzola • \\ Gerlando Quintini $\cdot$ Marianna Lo Re $\cdot$ Simona Talamanca • \\ Valeria Terruso • Marco D'Amelio • Giovanni Savettieri
}

Received: 21 February 2010/ Accepted: 24 November 2010

(C) Springer-Verlag 2010

\begin{abstract}
Thrombotic thrombocytopenic purpura (TTP) is an autoimmune disorder characterised by fever, microangiopathic haemolytic anaemia, renal insufficiency, and thrombocytopenia. Neurological involvement, a prominent component of TTP, is characterised by a variety of brain lesions which include reversible cerebral oedema or magnetic resonance imaging (MRI) features of reversible posterior leukoencephalopathy syndrome (RPLS). TTP is frequently associated with deficiency of the von Willebrand factor-cleaving protease, ADAMTS13.

Here, we report a case of TTP with severe acute encephalopathy. Posterior leukoencephalopathy and brainstem oedema with triventricular hydrocephalus were observed on MRI. The low activity of ADAMTS13 was not observed and ADAMTS-13 antibodies were absent. Neurological symptoms and patient's condition were completely resolved by plasma exchange therapy in addition to high dose of methylprednisolone.
\end{abstract}

Keywords Reversible posterior leukoencephalopathy · Thrombotic thrombocytopenic purpura · ADAMTS-13 . Plasma exchange

P. Aridon · P. Ragonese - M. A. Mazzola - M. Lo Re .

S. Talamanca · V. Terruso · M. D'Amelio - G. Savettieri ( $\square)$

Biomedicina Sperimentale e Neuroscienze Cliniche (BIONEC), University of Palermo, Via Gaetano La Loggia 1,

90129 Palermo, Italy

e-mail: gsavetti@tin.it

G. Quintini

U.O. di Ematologia, Azienda Ospedaliera Universitaria Policlinico Paolo Giaccone dell'Università degli Studi di Palermo, Palermo, Italy

\section{Introduction}

Posterior reversible leukoencephalopathy syndrome (RPLS) is a recently proposed clinico-neuroradiological entity presenting with a variety of neurological features [1]. These, although potentially devastating, are reversible on prompt diagnosis and appropriate treatment. Delayed diagnosis can lead to long-term neurological disability [2]. A relatively symmetrical pattern of involvement, typically in the subcortical white matter and occasionally in the cortex of the posterior circulation area of the brain is evident on magnetic resonance imaging (MRI) studies [1]. Common causes of RPLS are hypertensive encephalopathy, eclampsia, cyclosporin-A neurotoxicity, and uremic encephalopathy. Although the majority cases of RPLS are associated with hypertensive disorders, it has also been described in normotensive patients in association with thrombotic thrombocytopenic purpura (TTP) [3].

\section{Case report}

A previously healthy 53-year-old Caucasian man was admitted in an emergency department for the rapid onset of disturbances of gait, dizziness, urinary incontinence and lethargy. The pulse rate was 80 beats/min, temperature $38^{\circ} \mathrm{C}$ and blood pressure was $260 / 180 \mathrm{mmHg}$, partially decreased by administered nitroprusside, furosemide and clonidine.

White and red blood cells count and haemoglobin were normal $\left(7.5 \times 10^{3} / \mu 1,5.55 \times 10^{6} / \mu 1,15.7 \mathrm{~g} / \mathrm{dl}\right)$. Platelet count $\left(86.0 \times 10^{3} / \mu \mathrm{l}\right)$, LDH $(642 \mathrm{mg} / \mathrm{dl})$, serum creatinine $(1.90 \mathrm{mg} / \mathrm{dl})$, and urea $(53 \mathrm{mg} / \mathrm{dl})$ were out of normal range. A brain CT scan showed an enlargement of the lateral and third ventricles. The day after, the patient 
started complaining weakness in lower limbs, mental confusion and impaired attention and because of worsening of symptoms he was admitted to our department. He was lethargic and neurological examination showed hyperreflexia and pathological reflexes in all four limbs. An electroencephalogram (EEG) was characterised by marked slowness in the left posterior hemisphere without evidence of epileptic activity.

MRI of the brain revealed abnormal signal bilaterally in the white matter of cerebral and cerebellar hemispheres with the involvement of midbrain and cerebellar peduncles (Fig. 1a). A triventricular hydrocephalus, presumably caused by brainstem oedema, was observed.

A lumbar puncture revealed a limpid and uncoloured cerebrospinal fluid (CSF) with normal cell count (0.8/ $\mathrm{mm}^{3}$ ) and a barely elevated total protein concentration ( $77 \mathrm{mmg} / \mathrm{dl}$, normal value $<40 \mathrm{mg} / \mathrm{dl}$ ) with positive Pandy reaction. Polymerase chain reaction assay of Epstein-Barr virus (EBV)-DNA, Herpes simplex virus (HSV)-DNA,
Cytomegalovirus (CMV)-DNA was negative. CSF cultures had no bacterial, viral and fungal growth.

After 2 days from admission, laboratory findings revealed a platelet count of $58.0 \times 10^{3} / \mu 1$. Lactate dehydrogenase (LDH): $562 \mathrm{mg} / \mathrm{dl}$ (normal 200-480 mg/dl); serum creatinine: $3.04 \mathrm{mg} / \mathrm{dl}$ (normal $0.67-1.17 \mathrm{mg} / \mathrm{dl}$ ); urea: $106 \mathrm{mg} / \mathrm{dl}$ (normal $10-50 \mathrm{mg} / \mathrm{dl}$ ); bilirubin level: $1.42 \mathrm{mg} / \mathrm{dl}$ (normal $0.3-1.1 \mathrm{mg} / \mathrm{dl}$ ); AST: $38 \mathrm{U} / \mathrm{l}$ (max. $37 \mathrm{U} / \mathrm{l}$ ); GGT: $72 \mathrm{U} / 1$ (normal 8-61 U/l); rheumatoid factor: $106 \mathrm{IU} / \mathrm{ml}$; coagulation profile, complement $\mathrm{C} 3$ and C4: normal.

The results of serological tests for acute infection with cytomegalovirus, Epstein-Barr virus, Herpes simplex virus, and Herpes zoster virus were negative. Serological tests of antibodies to hepatitis $\mathrm{B}$ and $\mathrm{C}$ virus and human immunodeficiency virus were also negative.

Tests for serum antibodies, including anticardiolipin, perinuclear anti-neutrophil cytoplasmatic antibodies (pANCA), cytoplasmic anti-neutrophil cytoplasmatic antibodies
Fig. 1 T2-weighted axial and T1-weighted sagittal section MRI. a Two days after the admission to our department: hyperintensity in the bilateral white matter of cerebral and cerebellar hemispheres with involvement of midbrain and cerebellar peduncles, $\mathbf{b}$ after 2 weeks, c 3 months later: almost complete resolution of abnormal signal on MRI
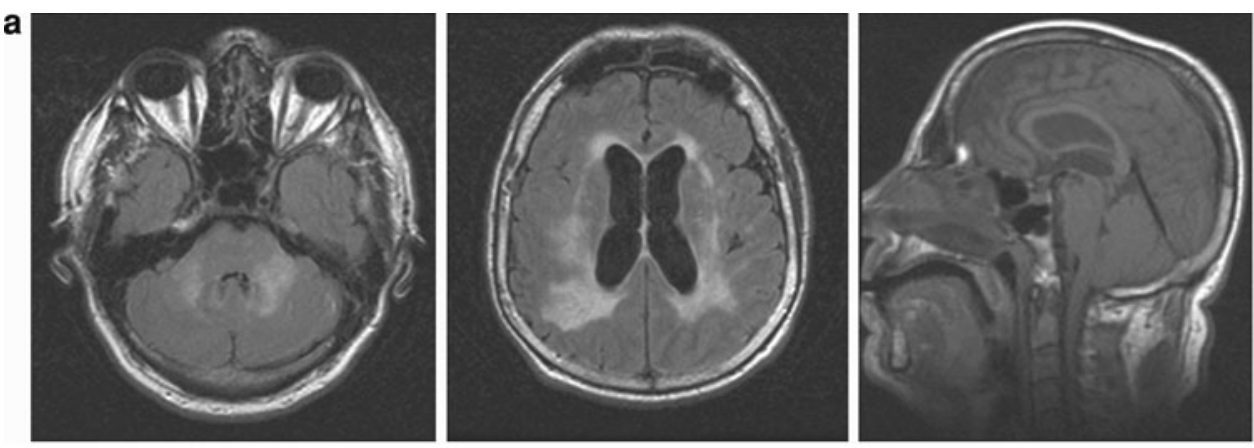

b
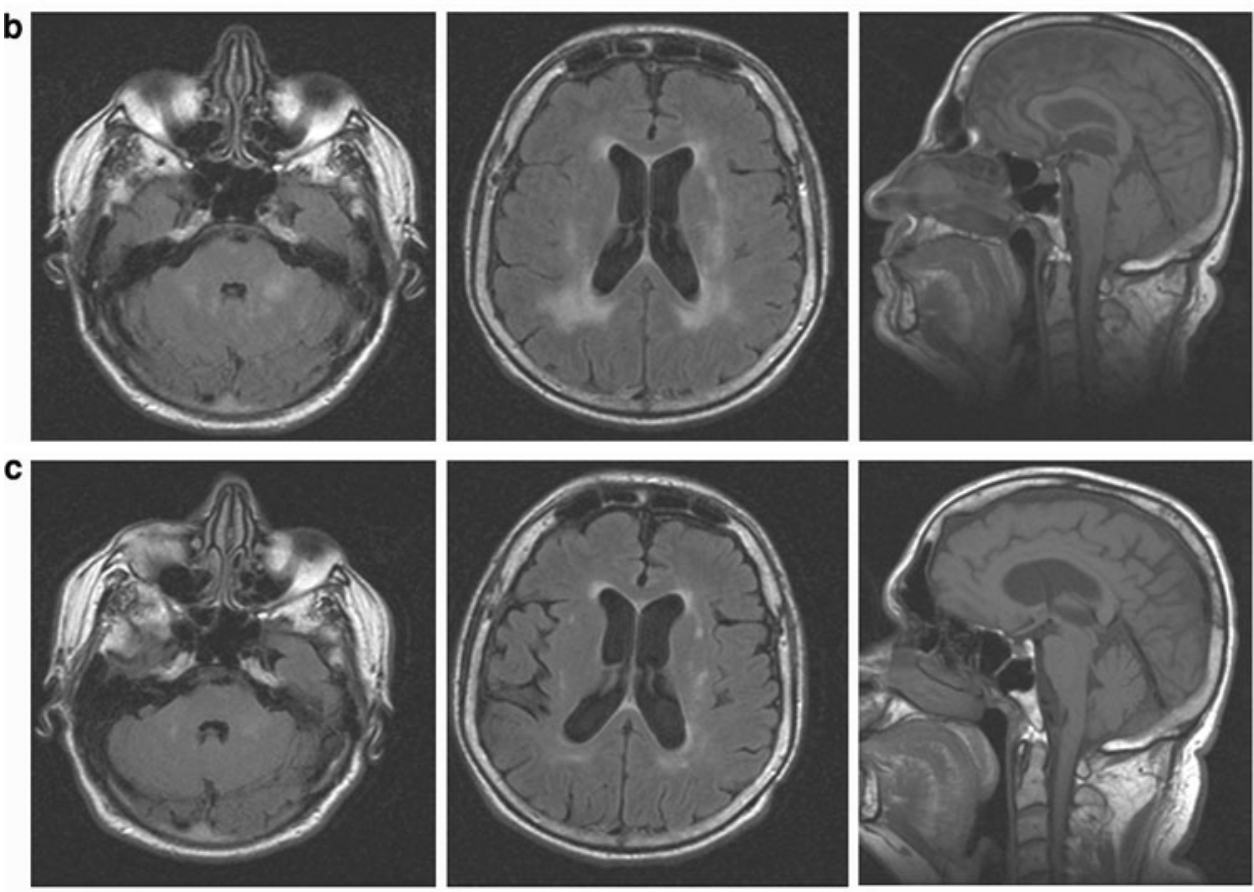

AX-T2

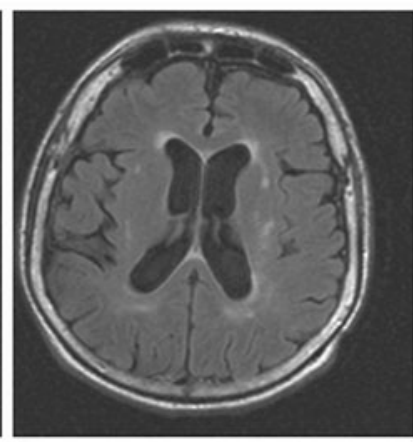

$\mathrm{AX}-\mathrm{T} 2$

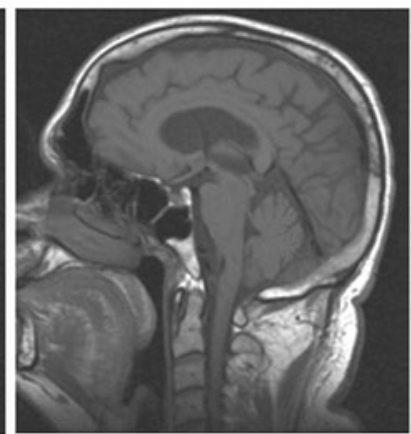

SAG-T1 
(cANCA), antinuclear antibody (ANA), anti-double stranded DNA antibody (dsDNA), anti-smooth muscle antibodies (anti-SM), anti-smooth muscle-ribonucleoprotein (anti-SM RNP), anti-Sjögren's syndrome A (anti-SSA) and antiSjögren's syndrome B (anti-SSB) were negative.

A 5-day regimen of high-dose intravenous administration of methylprednisolone (1 g/day) was started and within a week, an improvement of confusion and attentional difficulties was observed.

A diagnosis of RPLS in a patient with TTP was suggested by a second platelet count of $40.0 \times 10^{3} / \mu \mathrm{l}$, the presence of schistocytes in the peripheral blood smear, in addition to acute renal failure, confusion and other neurological symptoms, and MRI features. A blood sample of the patient was sent to a reference laboratory for ADAMTS-13 assays. The patient was transferred to the haematology division. More details regarding clinical and therapeutic aspects of this recovery are reported elsewhere [4]. Briefly, a prompt plasma exchange therapy was started, with daily exchanges of one plasma volume while steroid therapy was continued. After eight daily plasma exchange without complete remission, an additional immunosuppressive treatment with rituximab (at a standard dosage of $375 \mathrm{mg} / \mathrm{m}^{2}$ ) was administered weekly for 4 weeks. This treatment was continued despite ADAMTS-13 activity was $100 \%$ and no antibodies against ADAMTS-13 were detected. The platelet count was normalised and the neurological status improved progressively. The patient became totally normal after 3 months and a brain MRI showed nearly complete resolution of brainstem oedema and the T2 signal changes (Fig. 1c). Six months later, his neurological examination was normal. Twenty-four months later, no relapses have been observed and the patient is still healthy.

\section{Discussion and conclusion}

RPLS, also known as posterior reversible encephalopathy syndrome (PRES), is a clinical condition characterised by an acute or subacute onset of neurological symptoms (headache, altered mental status, confusion, and drowsiness), visual disturbance (hemianopsia, visual neglect, and cortical blindness), seizures associated with imaging findings of reversible cortical and subcortical vasogenic brain edema [5]. Eclampsia, hypertensive emergency, renal failure, autoimmune diseases and immunosuppressive therapy are common precipitating factors in RPLS [6].

The endothelial injury and the following release of factors promoting platelet activation and hyaline microthrombi formation in addition to blood-brain barrier injury are the most important pathophysiologic mechanisms for TTP-associated RPLS [3]. These factors could cause hypoperfusion leading to stroke or, on the contrary, cerebral micro-haemorrhages due to thrombocytopenia and hypertension [7].

We present a patient with TTP, elevated blood pressure and posterior vasogenic oedema on MRI consistent with posterior leukoencephalopathy. The course of these MRIfeatured lesions permitted us to define it as reversible.

Plasma exchange represents the first treatment of TTP patients with or without ADAMTS-13 deficiency. These two groups differ in the frequency of relapses. In fact, patients without ADAMTS-13 deficiency almost never relapse, on the contrary, about $50 \%$ of the patients with ADAMTS-13 deficiency suffer relapse within 2 years [8].

In our patient, in addition to the methylprednisolone, plasma exchange was started when the diagnosis of TTP was done, and as previously reported by other authors [9, 10], a rapid improvement of haemolysis and a normalization of platelet count were seen.

This case shows the importance of the recognition and prompt treatment of patient with TTP and further strengthens the evidence that, even if the demonstration of plasma level of ADAMTS-13 and its antibodies are diagnostic, the absence of this diagnostic test justify the beginning of therapy without waiting the test results.

Acknowledgments We thank the reviewers for their careful reading of the manuscript and helpful comments and Melanie Flynn for detailed and insightful corrections.

Conflict of interest The authors certify that there is no current or potential conflict of interest in relation to this article.

\section{References}

1. Lee VH, Wijdicks EF, Manno EM, Rabinstein AA (2008) Clinical spectrum of reversible posterior leukoencephalopathy syndrome. Arch Neurol 65:205-210

2. Pratap JN, Down JF (2008) Posterior reversible encephalopathy syndrome: a report of a case with atypical features. Anaesthesia 63:1245-1248

3. Bakshi R, Shaikh ZA, Bates VE, Kinkel PR (1999) Thrombotic thrombocytopenic purpura: brain CT and MRI findings in 12 patients. Neurology 52:1285-1288

4. Caramazza D, Quintini G, Abbene I, Coco LL, Malato A, Di Trapani R, Saccullo G, Pizzo G, Palazzolo R, Barone R, Mazzola G, Rizzo S, Ragonese P, Aridon P, Abbadessa V, Siragusa S (2010) Rituximab for managing relapsing or refractory patients with idiopathic thrombotiv thrombocytopenic purpura-haemolytic uraemic syndrome. Blood Transfus 8(3):203-210

5. Burrus TM, Wijdicks EF, Rabinstein AA (2009) Brain lesions are most often reversible in acute thrombotic thrombocytopenic purpura. Neurology 73:66-70

6. Pula JH, Eggenberger E (2008) Posterior reversible encephalopathy syndrome. Curr Opin Ophthalmol 19:479-484

7. Bas DF, Oguz KK, Topcuoglu MA (2008) Atypical reversible posterior leukoencephalopathy syndrome in thrombotic thrombocytopenic purpura. Intern Med 47(21):1931-1934 
8. Sadler JE (2008) Von Willebrand factor, ADAMTS13, and thrombotic thrombocytopenic purpura. Blood 112:11-18

9. Sarode R (2009) Atypical presentations of thrombotic thrombocytopenic purpura: a review. J Clin Apher 24:47-52
10. Brunskill SJ, Tusold A, Benjamin S, Stanworth SJ, Murphy MF (2007) A systematic review of randomized controlled trials for plasma exchange in the treatment of thrombotic thrombocytopenic purpura. Transfus Med 17:17-35 